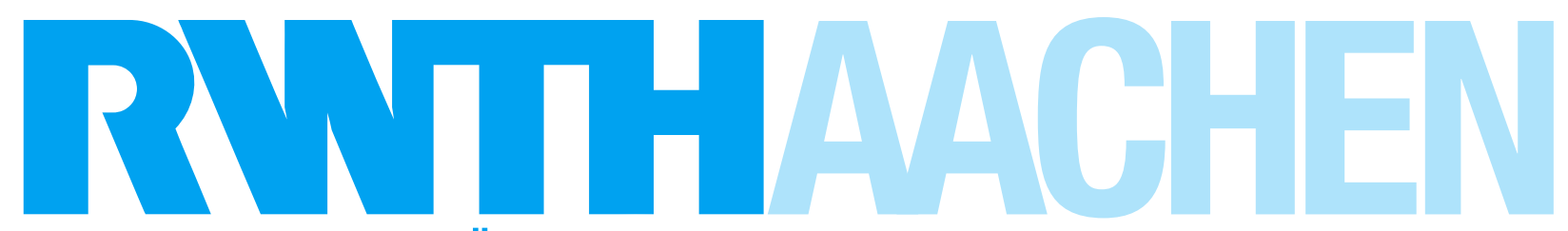

RHEINISCH-WESTFÄLISCHE TECHNISCHE HOCHSCHULE AACHEN Institut für Mathematik

Optimization problems for weighted

Sobolev constants

by

Catherine Bandle

Alfred Wagner

Report No. 7

2005

Dezember 2005

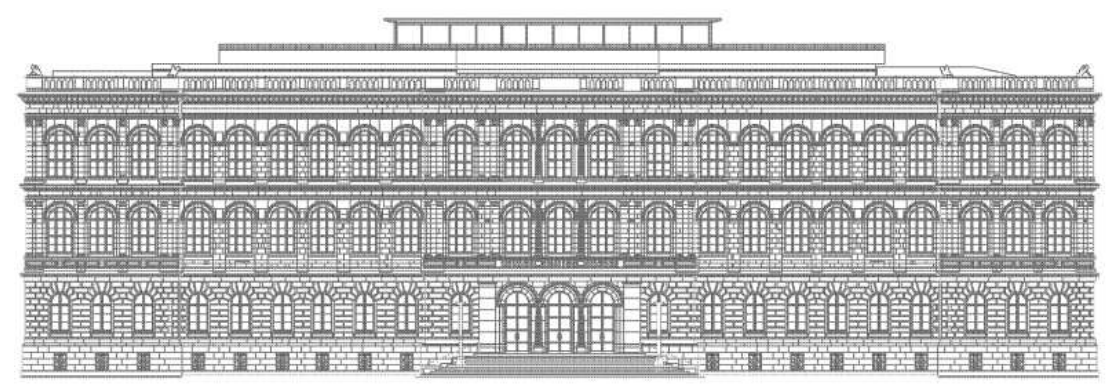

Institute for Mathematics, RWTH Aachen University

Templergraben 55, D-52062 Aachen

Germany 


\title{
Optimization problems for weighted Sobolev constants
}

\author{
Catherine Bandle*and Alfred Wagner ${ }^{\dagger}$
}

December 20, 2005

\begin{abstract}
In this paper we study a variational problem under a constraint on the mass. Using a penalty method we prove the existence of an optimal shape. It will be shown that the minimizers are Hölder continuous and that for a large class they are even Lipschitz continuous. Necessary conditions in form of a variational inequality in the interior of the optimal domain and a condition on the free boundary are derived.
\end{abstract}

Mathematics Subject Classification 2000: 49J20, 49K20, 35J65.

Keywords: calculus of variations, free boundaries, regularity of the minimizers.

\section{Introduction}

Let $D \in \mathbb{R}^{N}$ be a bounded domain and let $a(x)$ and $b(x)$ be positive, continuous functions in $D$. Consider for an arbitrary real number $p>1$ weighted Sobolev constants of the following form

$$
\begin{array}{r}
S_{p}(D)=\inf _{v} \int_{D} a(x)|\nabla v|^{p} d x, v \in \mathcal{K}(D) \text { where } \\
\mathcal{K}(D)=\left\{w \in W_{0}^{1, p}(D): w \geq 0, \int_{D} b(x) w d x=1\right\} .
\end{array}
$$

It follows from the Sobolev embedding theorem that there exists a minimizer $u$ which solves the Euler-Lagrange equation

$$
\operatorname{div}\left(a(x)|\nabla u|^{p-2} \nabla u\right)+S_{p}(D) b(x)=0 \text { in } D, u=0 \text { on } \partial D .
$$

*Mathematisches Institut, Universität Basel, Rheinsprung 21, CH-4051 Basel, Switzerland, email catherine.bandle@unibas.ch

${ }^{\dagger}$ Institut für Mathematik, RWTH Aachen, Templergraben 55, D-52062 Aachen Germany, e-mail wagner@instmath.rwth-aachen.de 
The first question addressed in this paper is to study the smallest value $s_{p}(m)$ of $S_{p}(D)$ when $D$ ranges among all domains contained in a fixed bounded domain $B \subset \mathbb{R}^{N}$, with prescribed measure $M(D):=\int_{D} b d x=m$. We are mainly interested in the existence of an optimal domain and the regularity of the minimizers.

For this purpose we follow a strategy used in [19] for eigenvalue problems. The idea which goes back to the pioneering papers of Alt and Caffarelli [1] and Alt, Caffarelli and Friedman [2], is to introduce a penalty term depending on $t$ and to consider a variational problem in $B$ without constraints. It has the advantage that it involves only the state function and not the optimal shape which is difficult to grasp.

Such a problem appeared for the first time in the literature in connection with the problem of the torsional rigidity of cylindrical beams. In this case $D$ is a simply connected domain in the plane, $p=2$ and $a(x)=b(x)=1$ and $B$ is a large circle such that $|B|>m$. It has been conjectured by St.Venant in 1856 and proved by Polyà cf. [15] that the optimal domain is the circle. The same questions have been studied in [6] for the special case $p=2$ and $a(x)=1$. A major ingredient there is the isoperimetric inequality which is not available for non constant $a(x)$. Many references and results concerning Sobolev constants with different types of weights can be found in [13] and [16]. For applications to boundary value problems cf. [4] and the references cited therein.

We shall assume that $a(x)$ and $b(x)$ meet the following assumptions:

(A1) $a(x), b(x) \in C^{0,1}(B)$;

(A2) there exist positive constants $a_{\min }$ and $a_{\max }$ such that $a_{\min } \leq a(x) \leq$ $a_{\max }$

(A3) there exists a positive constant $b_{\max }$ such that $0 \leq b(x) \leq b_{\max }$.

The plan of this paper is as follows. First we discuss the Sobolev constant $S_{p}(D)$ in multiply connected domains $D$. It turns out that it behaves differently from other similar quantities like the smallest eigenvalues. Then we prove the existence of a minimizer in $W_{0}^{1, p}(B)$ and of a corresponding optimal domain. The next chapter deals with the variational inequality which has to be satisfied by the minimizers and the characterization of the free boundary between their support and the region where they vanish. In the last chapter we prove regularity results for the minimizers, in particular the Lipschitz continuity.

\section{Qualitative properties}

In this section we list some general properties of $S_{p}(D)$. Instead of (1.1) it will sometimes be more convenient to use the equivalent form

$$
S_{p}(D)=\inf _{W_{0}^{1, p}(D)} \frac{\int_{D} a(x)|\nabla v|^{p} d x}{\left(\int_{D} b(x)|v| d x\right)^{p}} .
$$


Every minimizer is a multiple of $u$ where $u$ is the unique solution of

$$
\operatorname{div}\left(a(x)|\nabla u|^{p-2} \nabla u\right)+b(x)=0 \text { in } D, u=0 \text { on } \partial D .
$$

Lemma $1 S_{p}(D)$ is monotone with respect to $D$ in the sense that if $D_{1} \subset D_{2}$ then $S_{p}\left(D_{1}\right) \geq S_{p}\left(D_{2}\right)$.

Proof The assertion is an immediate consequence of the fact that every admissible function for $S_{p}\left(D_{1}\right)$, extended as 0 outside of $D_{1}$ is an admissible function for $S_{p}\left(D_{2}\right)$.

Lemma 2 Let $X$ and $Y$ be two domains in $\mathbb{R}^{N}$ such that $X \cap Y=\emptyset$. Then

$$
S_{p}(X \cup Y)^{-\frac{1}{p-1}}=S_{p}(X)^{-\frac{1}{p-1}}+S_{p}(Y)^{-\frac{1}{p-1}} .
$$

Proof Let $u_{X}$ and $u_{Y}$ be minimizers for $S_{p}(X)$ or $S_{p}(Y)$, resp. which are solutions of (2.2) in $X$ or $Y$, resp.. Consequently

$$
\begin{array}{r}
\int_{X} a\left|\nabla u_{X}\right|^{p} d x=\int_{X} b u_{X} d x=S_{p}^{-\frac{1}{p-1}}(X) \text { and } \\
\int_{Y} a\left|\nabla u_{Y}\right|^{p} d x=\int_{Y} b u_{Y} d x=S_{p}^{-\frac{1}{p-1}}(Y)
\end{array}
$$

Choosing as a test function in (2.1)

$$
v= \begin{cases}u_{X} & \text { in } X \\ u_{Y} & \text { in } Y\end{cases}
$$

we get

$$
S_{p}(X \cup Y) \leq \frac{1}{\left(S_{p}(X)^{-\frac{1}{p-1}}+S_{p}(Y)^{-\frac{1}{p-1}}\right)^{p-1}} .
$$

Let $u$ be a minimizer of $S_{p}(X \cup Y)$. Then keeping in mind that

$$
\int_{X} a|\nabla u|^{p} d x \geq S_{p}(X)\left(\int_{X} b u d x\right)^{p}, \int_{Y} a|\nabla u|^{p} d x \geq S_{p}(Y)\left(\int_{Y} b u d x\right)^{p},
$$

we find

$$
S_{p}(X \cup Y) \geq \frac{S_{p}(X)\left(\int_{X} b u d x\right)^{p}+S_{p}(Y)\left(\int_{Y} b u d x\right)^{p}}{\left(\int_{X} b u d x+\int_{Y} b u d x\right)^{p}} .
$$

Set $I:=\int_{X} b u d x+\int_{Y} b u d x, \int_{X} b u d x:=\lambda I$ and $\int_{Y} b u d x=(1-\lambda) I$. Then

$$
S_{p}(X \cup Y) \geq S_{p}(X) \lambda^{p}+S_{p}(Y)(1-\lambda)^{p}=: h(\lambda) .
$$

This function $h(\lambda)$ achieves its minimum for

$$
\lambda=\frac{S_{p}(Y)^{1 /(p-1)}}{S_{p}(X)^{1 /(p-1)}+S_{p}(Y)^{1 /(p-1)}} .
$$


Inserting this expression into $h(\lambda)$ we get

$$
S_{p}(X \cup Y) \geq \frac{1}{\left(S_{p}(X)^{-\frac{1}{p-1}}+S_{p}(Y)^{-\frac{1}{p-1}}\right)^{p-1}} .
$$

This together with (2.3) proves the assertion.

From this lemma we get immediately the estimate: If $S_{p}(X)<S_{p}(Y)$ then

$$
\frac{S_{p}(X)}{2^{p-1}} \leq S_{p}(X \cup Y) \leq \frac{S_{p}(Y)}{2^{p-1}} .
$$

Remark 1 Notice that the formula for $S_{p}(X \cup Y)$ in multiply connected domains differs from the one for the principal eigenvalue

$$
\lambda_{p}(D)=\inf _{W_{0}^{1, p}(D)} \frac{\int_{D} a|\nabla v|^{p} d x}{\int_{D} b|v|^{p} d x}
$$

In this case Lemma 2 has to be replaced by

$$
\lambda_{p}(X \cup Y)=\lambda_{p}(X), \text { where } \lambda_{p}(X) \leq \lambda_{p}(Y) .
$$

Definition 1 For all positive $M \leq M(B)$ set

$$
s_{p}(M):=\inf _{D} S_{p}(D) \text { where } D \in\left\{D^{\prime} \subset B: M\left(D^{\prime}\right) \leq M\right\}
$$

If for some region $D_{0}$ with measure $M s_{p}(M)=S_{p}\left(D_{0}\right)$, then $D_{0}$ is called optimal region for $s_{p}(M)$.

By Lemma 1 the infimum is the same if $D^{\prime}$ varies in the smaller class of domains with $M\left(D^{\prime}\right)=M$.

In the chapter on regularity we shall need the quantity

$$
\sigma_{p}=\inf _{(0, M(B))} M^{p+p / N-1} s_{p}(M) .
$$

The following lemma will be crucial for our considerations.

Lemma 3 Assume (A1),(A3) and the weaker form of (A2), namely (A2') $\quad 0<a_{\min } \leq a(x)$.

Then $\sigma_{p}>0$.

Proof We have

$$
S_{p}(D) \geq \frac{a_{\min }}{b_{\max }^{p}} \inf _{W_{0}^{1, p}(D)} \frac{\int_{D}|\nabla v|^{p} d x}{\left(\int_{D}|v| d x\right)^{p}} .
$$

Let

$$
T_{p}(D):=\inf _{W_{0}^{1, p}(D)} \frac{\int_{D}|\nabla v|^{p} d x}{\left(\int_{D}|v| d x\right)^{p}}
$$


If $D^{*}$ denotes the ball with the same volume as $D$ then by a symmetrization and a scaling argument we get

$$
T_{p}(D) \geq T_{p}\left(D^{*}\right)=\left(\frac{\left|B_{1}\right|}{|D|}\right)^{p+p / N-1} T_{p}\left(B_{1}\right)
$$

Hence

$$
\begin{gathered}
S_{p}(D) \geq \frac{a_{\min }}{b_{\max }^{p}}|D|^{1-p-p / N} c(N, p) \geq \frac{a_{\min }}{b_{\max }^{p}} b_{\min }^{p+p / N-1} M^{1-p-p / N} c(N, p), \\
\text { where } c(N, p):=\left|B_{1}\right|^{p+p / N-1} T_{p}\left(B_{1}\right),
\end{gathered}
$$

which implies that

$$
\sigma_{p} \geq \frac{b_{\min }^{p+p / N-1} a_{\min }}{b_{\max }^{p}} c(N, p)>0
$$

More results on $\sigma_{p}$ is found in [5].

\section{Existence}

Let $B \subset \mathbb{R}^{N}$ be a bounded fundamental domain, e.g. a large ball, and let $t>0, \epsilon>0$ be arbitrary fixed numbers. We consider the functional $J_{\epsilon, t}: W_{0}^{1, p}(B) \rightarrow \mathbb{R}^{+}$given by

$$
J_{\epsilon, t}(v):=\frac{\int_{B} a(x)|\nabla v(x)|^{p} d x}{\left(\int_{B} b(x)|v(x)| d x\right)^{p}}+f_{\epsilon}\left(\int_{\{v>0\}} b(x) d x\right),
$$

where

$$
f_{\epsilon}(s)=\left\{\begin{aligned}
\frac{1}{\epsilon}(s-t) & : \quad s \geq t \\
0 & : \quad s \leq t .
\end{aligned}\right.
$$

For $v \equiv 0$ we set $J_{\epsilon, t}(v)=\infty$. At first we are interested if the following variational problem has a minimizer

$$
\mathcal{J}_{\epsilon, t}=\inf _{\mathcal{K}} J_{\epsilon, t}(v)
$$

Lemma 4 Under the assumptions (A1)-(A3) there exists a function $u_{\epsilon} \in \mathcal{K}$ such that

$$
J_{\epsilon, t}(u)=\mathcal{J}_{\epsilon, t} .
$$

Proof Since the functional is bounded from below there exist minimizing sequences $\left\{u_{k}\right\}_{k \geq 1} \subset \mathcal{K}$. Assume that $J_{\epsilon, t}\left(u_{k}\right)<c_{0}$ for all $k$. We normalize $u_{k}$ such that

$$
\int_{B} b(x) u_{k}(x) d x=1 .
$$

Therefore $\int_{B} a\left|\nabla u_{k}(x)\right|^{p} d x<c_{0}$ and by [(A2)] also $\left\|\nabla u_{k} \mid\right\|_{L^{p}\left(B_{R}\right)}$ is uniformly bounded from above. Hence there exists a $u \in W_{0}^{1, p}(B)$ such that 
- $\nabla u_{k} \rightarrow \nabla u$ weakly in $L^{p}(B)$;

- $u_{k} \rightarrow u$ strongly in $L^{q}(B)$, for $q<n p /(N-p)$ if $p<N$ and for all $q \geq 1$ otherwise;

- $u_{k} \rightarrow u \in \mathcal{K}$ almost everywhere in $B$.

For the last statement see e.g. [Rudin] Theorem 3.12. This result implies in particular that $\int_{B} u(x) b(x) d x=1$. Since $\left\{\int_{B} a(x)|\nabla u(x)|^{p} d x\right\}^{1 / p}$ is a norm in $W_{0}^{1, p}(B)$ and since norms are lower semicontinuous with respect to weak convergence, the inequality

$$
\int_{B} a(x)|\nabla u(x)|^{p} d x \leq \liminf _{k \rightarrow \infty} \int_{B} a(x)\left|\nabla u_{k}(x)\right|^{p} d x
$$

holds. Moreover since $u_{k} \rightarrow u$ in $L^{1}\left(B_{R}\right)$ we have $\int_{B_{R}} b u d x=1$.

Let $\chi_{\left\{u_{k}>0\right\}}$ denote the characteristic function of the support of $u_{k}$, i.e.

$$
\chi_{\left\{u_{k}>0\right\}}(x)= \begin{cases}1 & \text { if } u_{k}(x)>0 \\ 0 & \text { otherwise }\end{cases}
$$

Next we want to prove that

$$
\int_{B_{R}} \chi_{\{u>0\}}(x) b(x) d x \leq \liminf _{k \rightarrow \infty} \int_{B_{R}} \chi_{\left\{u_{k}>0\right\}}(x) b(x) d x .
$$

We have, denoting by $|G|$ the Lebesgue measure of a set $G$,

$$
\begin{aligned}
& \int_{B}\left|\chi_{\left\{u_{k}>0\right\}}(x)-\chi_{\{u\}}(x)\right| b(x) d x \\
& \leq b_{\max }\left|\{u>0\} \backslash\left\{u_{k}>0\right\} \cup\left\{u_{k}>0\right\} \backslash\{u>0\}\right| \\
& \leq\left|\left\{x:\left|u(x)-u_{k}(x)\right|>\delta\right\}\right|+b_{\max }\{\mid x: 0<u(x)<\delta\}\left|+b_{\max }\left\{\mid x: 0<u_{k}(x)<\delta\right\}\right|
\end{aligned}
$$

for all $\delta>0$. Since $u_{k} \rightarrow u$ a.e. it follows that

$$
\left|\left\{x:\left|u(x)-u_{k}(x)\right|>\delta\right\}\right| \rightarrow 0 \text { as } k \rightarrow \infty .
$$

By the continuity of the Lebesgue measure

$$
|\{\mid x: 0<u(x)<\delta\}| \text { and }\left|\left\{\mid x: 0<u_{k}(x)<\delta\right\}\right| \rightarrow 0 \text { as } \delta \rightarrow 0 .
$$

Hence

$$
\begin{aligned}
& \liminf _{k \rightarrow \infty} \int_{B} \chi_{\left\{u_{k}>0\right\}}(x) b(x) d x \geq-\lim _{k \rightarrow \infty} \int_{B}\left|\chi_{\left\{u_{k}>0\right\}}(x)-\chi_{\{u\}}(x)\right| b(x) d x \\
& +\int_{B} \chi_{u>0}(x) b(x) d x
\end{aligned}
$$


This together with (3.2) implies

$$
J_{\epsilon, t}(u) \leq \inf _{\mathcal{K}} J_{\epsilon, t}(v)
$$

which completes the proof of the lemma.

In general, the minimizer $u$ of $\mathcal{J}_{\epsilon, t}$ depends on $\epsilon$. If we want to emphasize the $\epsilon$ - dependence we shall write $u_{\epsilon}$.

Open problem We expect that for $\epsilon$ sufficiently small, $u_{\epsilon}$ is independent of $\epsilon$ and $\mathcal{J}_{\epsilon, t}=\mathcal{J}_{\epsilon, 0}=s_{p}(t)$ for all $\epsilon \leq \epsilon_{0}$.

For simplicity we shall also use the following notation: for any $w \in \mathcal{K}$ set

$$
D_{w}:=\{w(x)>0\}, M_{w}:=\int_{D_{w}} b(x) d x .
$$

Theorem 1 There exists $u_{0} \in \mathcal{K}$ such that for every positive $t \leq M(B)$

$$
S_{p}\left(D_{u_{0}}\right)=s_{p}(t)
$$

Proof Let $D^{\prime} \subset B$ be an arbitrary domain in $B$ such that $\int_{M\left(D^{\prime}\right)} b d x=t$. Let $w \in W_{0}^{1, p}\left(D^{\prime}\right)$ be a minimizer of $S_{p}\left(D^{\prime}\right)$ with $w \equiv 0$ in $B \backslash D^{\prime}$ and let $u_{\epsilon}$ be a minimizer of $\mathcal{J}_{\epsilon, t}$ such that $\int_{B} b u_{\epsilon} d x=1$. Then $J_{\epsilon, t}\left(u_{\epsilon}\right) \leq J_{\epsilon, t}(w)$, i.e.

$$
\int_{B} a\left|\nabla u_{\epsilon}\right|^{p} d x+f_{\epsilon}\left(M_{u_{\epsilon}}-t\right) \leq S_{p}\left(D^{\prime}\right)
$$

Then $\int_{B} a\left|\nabla u_{\epsilon}\right|^{p} d x$ and by the assumption (A3) also $\int_{B}\left|\nabla u_{\epsilon}\right|^{p} d x$ are bounded from above by a constant which is independent of $\epsilon$. Therefore there exists a subsequence $u_{\epsilon}$ such that

$$
u_{\epsilon} \rightarrow u_{0} \text {, weakly in } W_{0}^{1, p}(B), \quad u_{\epsilon} \rightarrow u_{0} \text { strongly in } L^{1}(B) \text { as } \epsilon \rightarrow 0 .
$$

Moreover $u_{0} \in \mathcal{K}$ and by the same arguments as in Lemma 4 we conclude that $M_{u_{0}} \leq t$. This together with Fatou's lemma implies

$$
\frac{\int_{B} a\left|\nabla u_{0}\right|^{p} d x}{\left(\int_{B} b u_{0} d x\right)^{p}}=\int_{B} a\left|\nabla u_{0}\right|^{p} d x \leq S_{p}\left(D^{\prime}\right) .
$$

Since this inequality holds for any $D^{\prime}$ with $M\left(D^{\prime}\right)=t$, the assertion follows.

\section{Necessary conditions}

\subsection{First variation}

Theorem 2 Let $u_{\epsilon}$ be a minimizer of $\mathcal{J}_{\epsilon, t}$ which is normalized such that $\int_{B} b(x) u_{\epsilon}(x) d x=1$. Then for all nonnegative smooth functions $\varphi$ with compact 
support in $B$, the following inequality holds:

$$
\begin{array}{r}
\int_{B} a(x)\left|\nabla u_{\epsilon}(x)\right|^{p-2} \nabla u_{\epsilon} \nabla \varphi(x) d x \leq \lambda \int_{B} b(x) \varphi(x) d x, \\
\text { where } \lambda:=\int_{B} a(x)\left|\nabla u_{\epsilon}(x)\right|^{p} d x .
\end{array}
$$

Proof For short we shall write $u$ instead of $u_{\epsilon}$. Since $u$ is a minimizer we have $\mathcal{J}_{\epsilon}(u) \leq \mathcal{J}_{\epsilon}\left((u-\delta \varphi)^{+}\right)$for every $\delta>0$ and $\varphi>0$. Set $v:=(u-\delta \varphi)^{+}$ and note that $D_{v} \subset D_{u}$. Hence by the monotonicity of $f_{\epsilon}(t)$ we have

$$
f_{\epsilon}\left(M_{u}\right) \geq f_{\epsilon}\left(M_{v}\right)
$$

and thus

$$
\frac{\int_{B} a(x)|\nabla u(x)|^{p} d x}{\left(\int_{B} b(x) u(x) d x\right)^{p}} \leq \frac{\int_{B} a(x)|\nabla v(x)|^{p} d x}{\left(\int_{B} b(x) v(x) d x\right)^{p}} .
$$

Using the normalization we get

$$
0 \leq \int_{B} a(x)|\nabla v(x)|^{p} d x-\int_{B} a(x)|\nabla u(x)|^{p} d x\left(\int_{B} b(x) v(x) d x\right)^{p} .
$$

We now discuss the integrals in more detail. Keeping in mind that $\int_{B} b u d x$ and $\int_{B} b \varphi d x$ are bounded we find, setting

$$
\begin{aligned}
I_{0} & :=\int_{B \cap\{u>\delta \varphi\}} b(x) u(x) d x, \\
\left(\int_{B} b(x) v(x) d x\right)^{p} & =\left(\int_{B \cap\{u>\delta \varphi\}} b(x)(u-\delta \varphi)(x) d x\right)^{p} \\
& =I_{0}^{p}-p \delta I_{0}^{p-1} \int_{B \cap\{u>\delta \varphi\}} b(x) \varphi(x) d x+o(\delta) .
\end{aligned}
$$

Next we compute

$$
\begin{array}{r}
\int_{B} a(x)|\nabla v(x)|^{p} d x=\int_{B \cap\{u>\delta \varphi\}} a(x)|\nabla(u-\delta \varphi)(x)|^{p} d x= \\
\int_{B \cap\{u>\delta \varphi\}} a(x)|\nabla u(x)|^{p} d x-p \delta \int_{B \cap\{u>\delta \varphi\}} a(x)|\nabla u(x)|^{p-2} \nabla u \nabla \varphi d x+\eta .
\end{array}
$$


The remainder term $\eta$ contains expressions of the form $\delta^{2} \int_{B}(x) a(x)|\nabla u|^{q} \tilde{\varphi} d x$ with $q \leq p-1$. Since $\int_{B} a|\nabla u|^{p} d x$ and $|\tilde{\varphi}|_{\infty}$ are bounded, we have

$$
\eta=O\left(\delta^{2}\right) \text {. }
$$

Plugging these expression into inequality (4.2) we get

$$
\begin{aligned}
& 0 \leq \int_{\{u>\delta \varphi\}} a(x)|\nabla u(x)|^{p} d x-p \delta \int_{\{u>\delta \varphi\}} a(x)|\nabla u(x)|^{p-2} \nabla u \nabla \varphi(x) d x- \\
& \int_{B} a|\nabla u|^{p} d x\left(I_{0}^{p}-p \delta I_{0}^{p-1} \int_{\{u>\delta \varphi\}} b(x) \varphi(x) d x\right)+o(\delta) .
\end{aligned}
$$

Observing that

$$
\int_{\{u>\delta \varphi\}} b(x) u(x) d x=1-\int_{\{u \leq \delta \varphi\}} b(x) u(x) d x,
$$

we deduce from (4.3) that

$$
\begin{aligned}
& p \delta \int_{\{u>\delta \varphi\}} a(x)|\nabla u(x)|^{p-2} \nabla u \nabla \varphi(x) d x \\
& \quad \leq \int_{B} a|\nabla u|^{p} d x\left\{p \int_{\{u \leq \delta \varphi\}} b u d x+p \delta \int_{\{u>\delta \varphi\}} b \varphi d x\right\}+o(\delta) .
\end{aligned}
$$

The expression in the brackets at the right-hand side of this inequality is bounded from above by

$$
p \delta \int_{B} b \varphi d x
$$

Hence we obtain, dividing by $p \delta>0$ and then letting $\delta$ tend to 0

$$
\int_{B} a(x)|\nabla u(x)|^{p-2} \nabla u \nabla \varphi(x) d x \leq \int_{B} a(x)|\nabla u(x)|^{p} d x \int_{B} b(x) \varphi(x) d x .
$$

This proves the theorem.

Corollary 1 In the interior of $D_{u_{\epsilon}}$, every normalized minimizer $u_{\epsilon}$ satisfies the Euler-Lagrange equation

$$
\operatorname{div}\left(a(x)\left|\nabla u_{\epsilon}(x)\right|^{p-2} \nabla u_{\epsilon}(x)\right)+\lambda b(x)=0 .
$$

$x_{0}$ be an inner point in $\left\{u_{\epsilon}>0\right\}$ and suppose that the ball $B_{\rho}\left(x_{0}\right)$ centered at $x_{0}$ satisfies $\overline{B_{\rho}\left(x_{0}\right)} \subset\left\{u_{\epsilon}>0\right\}$. Let $\varphi \in C_{0}^{\infty}\left(B_{\rho}\left(x_{0}\right)\right)$. Choose $\delta$ so small that $v:=u+\delta \varphi>0$ in $B_{\rho}\left(x_{0}\right)$. The same arguments as in the previous theorem imply that

$$
\int_{B_{\rho}\left(x_{0}\right)} a|\nabla u|^{p-2} \nabla u \nabla \varphi d x=\gamma \int_{B_{\rho}\left(x_{0}\right)} b \varphi d x
$$

This proves the assertion.

Remark The proof of the previous Theorem holds also for $u_{0}$ which is the minimizer corresponding to $s_{p}(M)$ [cf. Theorem 1]. 


\subsection{Boundary condition}

We derive a necessary condition for the minimizers $u_{\epsilon}$ which has to be satisfied on $\partial D_{u_{\epsilon}}$ where it is smooth.

Theorem 3 Let $u_{\epsilon}$ be a minimizer of $\mathcal{J}_{t, \epsilon}$. On the smooth parts of $\partial D_{u}$, the following identity holds

$$
a(x)|\nabla u(x)|^{p}=\text { const.b(x) for } x \in \partial D_{u} .
$$

Proof Consider the function

$$
\tilde{u}(x):=u(x+\delta \eta(x)) .
$$

$\eta$ denotes a smooth vector field in $B$ with compact support satisfying the additional constraint

$$
\int_{\partial D_{u}} b(x) \eta(x) \cdot \nu(x) d S=0 .
$$

$\delta$ denotes a positive constant which is chosen so small, such that $x+\delta \eta(x) \in B$ for all $x \in B$. A consequence of this setting is

Lemma 5 Let $\eta$ be defined as above. Then

$$
\int_{D_{\tilde{u}}} b(x) d x=\int_{D_{u}} b(x) d x+o(\delta) .
$$

The claim follows by direct computation. We set $y=x+\delta \eta(x)$. Then $d x=(1-\delta \operatorname{div} \eta) d y+o(\delta)$. Hence we get because of (4.6).

$$
\begin{aligned}
\int_{D_{\tilde{u}}} b(x) d x & =\int_{D_{u}} b(y-\delta \eta)(1-\delta \operatorname{div} \eta) d y+o(\delta)=\int_{D_{u}} b(y) d y-\delta \int_{D_{u}} b \operatorname{div} \eta d y \\
& -\delta \int_{D_{u}} \eta \cdot \nabla b d y+o(\delta)=\int_{D_{u}} b(y) d y+o(\delta) .
\end{aligned}
$$

This proves the lemma.

By our assumption

$$
J_{\epsilon, t}(u) \leq J_{\epsilon, t}(\tilde{u}) .
$$

We expand the right hand side with respect to $\delta$ and get

$$
\begin{aligned}
\int_{D_{\tilde{u}}} a(x)|\nabla \tilde{u}(x)|^{p} d x & =\int_{D_{u}} a|\nabla u|^{p} d y-\delta \int_{D_{u}} a|\nabla u|^{p} \operatorname{div} \eta d y-\delta \int_{D_{u}} \eta \cdot \nabla a|\nabla u|^{p} d y \\
& +\delta p \int_{D_{u}} a|\nabla u|^{p-2} \nabla u \cdot D \eta \cdot \nabla u d y+o(\delta) .
\end{aligned}
$$


We integrate by parts, making use the smoothness of $\partial D_{u}$, and we obtain

$$
\begin{aligned}
\int_{D_{u}} a(x)|\nabla \tilde{u}(x)|^{p} d x & =\int_{D_{u}} a|\nabla u|^{p} d y-\delta p \int_{D_{u}} \operatorname{div}\left(a|\nabla u|^{p-2} \nabla u\right) \eta \cdot \nabla u d y \\
& +\delta(p-1) \int_{\partial D_{u}} a|\nabla u|^{p} \eta \cdot \nu d S+o(\delta) .
\end{aligned}
$$

Similarly we have

$$
\int_{D_{\tilde{u}}} b(x) \tilde{u}(x) d x=\int_{D_{u}} b(y) u(y) d y-\delta p \int_{D_{u}} b \eta \cdot \nabla u d y+o(\delta) .
$$

Inserting the above expansions into (4.8) rearranging terms we get for $\delta \rightarrow 0$ :

$$
\int_{\partial D_{u}} a|\nabla u|^{p} \eta \cdot \nu d S=0
$$

The equality comes from the fact that $\eta \cdot \nu$ can have any sign and the penalization term drops out by monotonicity. Because of (4.6) this implies

$$
a(x)|\nabla u(x)|^{p}=\text { const.b(x) for } x \in \partial D_{u} .
$$

\section{Regularity}

This section is devoted to the regularity of the minimizers $\mathcal{J}_{\epsilon, t}$. The notation will be the same as in the last section. If $p \geq N$ it follows immediately from the embedding theorems that the minimizers are Hölder continuous.

Theorem 4 Every solution $u$ of (4.1) belongs to $L^{\infty}(D)$ and satisfies

$$
|u|_{\infty} \leq\left(\frac{\lambda}{\sigma_{p}}\right)^{\frac{1}{p-1+p / N}} \frac{p+N p-N}{p},
$$

provided $\int_{B} b u d x=1$.

Proof By testing (4.1) with $(u-t)_{+}$we obtain, setting $D(t):=\{x \in D$ : $u(x)>t\}$ and $M(t):=M(D(t))$,

$$
\int_{D(t)} a|\nabla u|^{p} d x \leq \lambda \int_{D(t)} b(u-t) d x
$$

Using the fact that $\sigma_{p}>0$ we have

$$
\sigma_{p}\left(\int_{D(t)}(u-t) b d x\right)^{p} M^{1-\frac{p}{N}-p}(t) \leq \int_{D(t)} a|\nabla u|^{p} d x
$$


This together with (5.1) implies

$$
\sigma_{p}\left(\int_{D(t)}(u-t) b d x\right)^{p} M^{1-\frac{p}{N}-p}(t) \leq \lambda \int_{D(t)} b(u-t) d x .
$$

Integration by parts yields

$$
\int_{D(t)}(u-t) b d x=\int_{t}^{\infty} M(s) d s=: \hat{M}(t) .
$$

Inserting this expression into (5.2) we get

$$
\left(\frac{\sigma_{p}}{\lambda}\right)^{\frac{1}{p+p / N-1}} \leq-\hat{M}^{\prime} \hat{M}^{-\frac{p-1}{p+p / N-1}}
$$

Put for short $\gamma=\left(\frac{\sigma_{p}}{\lambda}\right)^{\frac{1}{p-1+p / N}}$ and $\alpha=\frac{p-1}{p+p / N-1}$. Since $\hat{M}(0)=1$ we find after integration

$$
\gamma(1-\alpha) t \leq 1-\hat{M}(t)^{1-\alpha}
$$

Hence

$$
t \leq \frac{1}{(1-\alpha) \gamma}=\left(\frac{\lambda}{\sigma_{p}}\right)^{\frac{1}{p+p / N-1}} \frac{p+N p-N}{p} .
$$

This establishes the assertion.

Next we we shall prove the Hölder continuity of the minimizers. For this purpose we need an additional assumption on $b$.

(A4) Let $\epsilon_{0}>0$ be an arbitrary fixed constant. Then there exist positive constants $c_{0}$ and $c_{1}<N$ such that for all $1 \leq \mu \leq 1+\epsilon_{0}$ and for all $x \in B$

$$
-c_{0}(1-\mu) \leq b\left(\frac{x}{\mu}\right)-b(x) \leq c_{1}(1-\mu) b(x) .
$$

Remark The condition (A4) holds for all polynomials. In the sequel $c$ denotes a constant which is independent of $R$.

Theorem 5 Let $B$ be convex. Assume (A1)-(A4) and $1<p<\infty$. Let $u \in \mathcal{K}$ be any minimizer of $s(M)$. Then $u \in C_{l o c}^{0, \beta}(B)$ for all $0 \leq \beta<1$.

The proof is done in several steps. Let us first collect some useful auxiliary result.

Our arguments rely heavily on a lemma of Morrey [14].

Lemma 6 (Morrey's Dirichlet growth theorem). Let $u \in W^{1, p}(B), 1<p<$ $N$. Suppose that there exist constants $0<c<\infty$ and $\beta \in(0,1]$ such that for all balls $B_{R}\left(x_{0}\right) \subset B$

$$
\int_{B \cap B_{R}\left(x_{0}\right)}|\nabla u|^{p} d x \leq c R^{N-p+\beta p}
$$

then $u \in C^{0, \beta}(B)$. 
In order to apply the above lemma we shall also need

Lemma 7 Let $\phi(t)$ be a nonnegative and nondecreasing function. Suppose that

$$
\phi(\rho) \leq a\left[\left(\frac{\rho}{R}\right)^{\alpha}+\delta\right] \phi(R)+b R^{\beta}
$$

for all $0 \leq \rho \leq R \leq R_{0}$, where $a, b, \alpha$ and $\beta$ are positive constants with $\beta<\alpha$. Then there exist positive constants $\delta_{0}=\delta_{0}(a, \alpha, \beta)$ and $c=C(a, \alpha, \beta)$ such that if $\delta<\delta_{0}$, then

$$
\phi(\rho) \leq c\left(\frac{\rho}{R}\right)^{\beta}\left[\phi(R)+b R^{\beta}\right]
$$

for all $0 \leq \rho \leq R \leq R_{0}$.

For the proof of this Lemma we refer to [11], Lemma 2.1 in Chapter III. Next we construct a comparison function for the functional $s(M)$ which will play an important role in the proof of the Hölder and Lipschitz continuity of $u$. Let $u$ be a minimizer of $s(M)$. Let $B_{R}\left(x_{0}\right)$ any ball of radius $R$ centered at $x_{0}$ such that $B_{2 R}\left(x_{0}\right) \subset B$. Set

$$
v(x)= \begin{cases}\hat{v}(x) & \text { if } x \in B_{R}\left(x_{0}\right) \\ u(x) & \text { if } x \in D_{u} \backslash B_{R}\left(x_{0}\right) \\ 0 & \text { otherwise }\end{cases}
$$

where $\hat{v}$ is the solution of

$$
\begin{aligned}
\operatorname{div}\left(a|\nabla \hat{v}|^{p-2} \nabla \hat{v}\right)+\lambda b(x) & =0 \text { in } B_{R}\left(x_{0}\right), \hat{v}=u \text { on } \partial B_{R}\left(x_{0}\right), \\
\lambda & =\int_{B} a|\nabla u|^{p} d x .
\end{aligned}
$$

Since

$$
\operatorname{div}\left(a|\nabla u|^{p-2} \nabla u\right)+\lambda b(x) \geq 0 \text { in } B_{R}\left(x_{0}\right),
$$

the maximum principle gives $\hat{v} \geq u$ in $B_{R}\left(x_{0}\right)$. Also observe that

$$
\int_{B_{R}\left(x_{0}\right)} a|\nabla \hat{v}|^{p} d x \leq \int_{B_{R}\left(x_{0}\right)} a|\nabla u|^{p} d x+\lambda p \int_{B_{R}\left(x_{0}\right)} b(\hat{v}-u) d x .
$$

Since $\hat{v}>0$ in $B_{R}\left(x_{0}\right)$ by the strong maximum principle (see e.g. [18]), we have $D_{u} \subseteq D_{v}$. Hence in general $v(x)$ cannot be used as a test function in the variational principle for $s(M)$. We therefore define $w(x):=v(\mu x)$ and choose $\mu \geq 1$ such that $M_{w}=M_{u}=M$. Since $B$ is convex, $D_{w} \subset B$ and $w(x)$ can be used as a test function of the variational characterization of $s(M)$. In the sequel we shall frequently use the notation

$$
\left.N_{u}:=\{u=0\} \cap B\right\}
$$

The following elementary estimate will be needed later on. 
Proposition 1 Let $u$ be a minimizer and let $v$ and $\mu$ be defined as above. Under the assumption (A4) the following estimates holds true for $R \leq R_{0}\left(\epsilon_{0}\right)$

$$
1 \leq \mu \leq 1+\frac{b_{\max }}{N-c_{1}} \frac{\left|N_{u} \cap B_{R}\left(x_{0}\right)\right|}{M} .
$$

Proof To simplify notation we write $B_{R}$ instead of $B_{R}\left(x_{0}\right)$. For $\tilde{\mu} \geq 1$ set

$$
g(\tilde{\mu}):=\tilde{\mu}^{-N} \int_{D_{v}} b\left(\frac{x}{\tilde{\mu}}\right) d x .
$$

By definition we have

$$
g(\mu)=\int_{D_{w}} b(x) d x=\int_{D_{u}} b(x) d x=M .
$$

By (A4)

$$
\begin{aligned}
g(\tilde{\mu}) & \leq \tilde{\mu}^{-N}\left(1+c_{1}(1-\tilde{\mu})\right) \int_{D_{v}} b(x) d x \\
& \leq \tilde{\mu}^{-N}\left(1+c_{1}(1-\tilde{\mu})\right)\left\{\int_{D_{u}} b(x) d x+b_{\max }\left|N_{u} \cap B_{R}\right|\right\} \\
& =\tilde{\mu}^{-N}\left(1+c_{1}(1-\tilde{\mu})\right) M\left(1+\frac{b_{\max }\left|N_{u} \cap B_{R}\right|}{M}\right) .
\end{aligned}
$$

If we evaluate the expression above at

$$
\tilde{\mu}_{0}=1+c \frac{\left.\left|N_{u} \cap B_{R}\right|\right\}}{M}=: 1+c \eta
$$

we obtain

$$
g\left(\tilde{\mu}_{0}\right) \leq M \tilde{\mu}_{0}^{-N}\left(1+\left(c c_{1}+b_{\max }\right) \eta+O\left(\eta^{2}\right)\right) \text { as } \eta \rightarrow 0 .
$$

Choosing $c=\frac{b_{\max }}{N-c_{1}}$ we find for $R$ sufficiently small that $g\left(\tilde{\mu}_{0}\right)<M$. This together with $g(1)>M$ proves the assertion.

Lemma 8 Let $u \in \mathcal{K}$ be any minimizer of $s(M)$ and let $\hat{v}$ and $\mu$ be defined as above. Then

$$
\int_{B_{R}\left(x_{0}\right)} a(x)|\nabla(u-\hat{v})|^{p} d x \leq c R^{(N-p)\left(1-\frac{p}{2}\right)}\left|N_{u} \cap B_{R}\left(x_{0}\right)\right|^{\frac{p}{2}}
$$

for $1<p \leq 2$ and

$$
\int_{B_{R}\left(x_{0}\right)} a(x)|\nabla(u-\hat{v})|^{p} d x \leq c\left|N_{u} \cap B_{R}\left(x_{0}\right)\right|
$$

for $p \geq 2$. 
Proof By definition we have, with $w(x)=v(\mu x)$ as above,

$$
s(M) \leq \frac{\int_{B} a|\nabla w|^{p} d x}{\left(\int_{B} b w d x\right)^{p}} .
$$

Observe that

$$
\int_{B} a|\nabla w|^{p} d x=\mu^{p-N} \int_{D_{v}} a_{\mu}|\nabla v|^{p} d x
$$

and

$$
\int_{B} b w d x=\mu^{-N} \int_{D_{v}} b_{\mu} v d x
$$

where $a_{\mu}(x)=a\left(\frac{x}{\mu}\right)$ and $b_{\mu}(x)=b\left(\frac{x}{\mu}\right)$. From (5.8) and the definition of $w$ it then follows that

$$
s(M) \mu^{N-p-N p}\left(\int_{D_{v}} b_{\mu} v d x\right)^{p} \leq \int_{D_{v}} a_{\mu}|\nabla v|^{p} d x .
$$

We write $D_{v}=D_{u} \backslash B_{R} \cup B_{R}$ and get, using (A4)

$$
\begin{aligned}
\int_{D_{v}} b_{\mu} v d x & =\int_{D_{v} \backslash B_{R}} b_{\mu} v d x+\int_{B_{R}} b_{\mu} \hat{v} d x \\
& =\int_{D_{u}} b_{\mu} u d x+\int_{B_{R}} b_{\mu}(\hat{v}-u) d x \\
& =\int_{D_{u}} b u d x+\int_{D_{u}}\left(b_{\mu}-b\right) u d x+\int_{B_{R}} b_{\mu}(\hat{v}-u) d x \\
& \geq \int_{D_{u}} b u d x-c(\mu-1) .
\end{aligned}
$$

For the last inequality we used the boundedness of $u$ (cf. Theorem 4 and the fact that $\hat{v} \geq u$ in $B_{R}$. Proposition 1 together with $\int_{D_{u}} b u d x=1$ then implies

$$
\int_{D_{v}} b_{\mu} v d x \geq 1+O\left(\left|N_{u} \cap B_{R}\right|\right)
$$

In order to estimate the right hand of (5.9) side we use the Lipschitz continuity of $a$ and obtain

$$
\begin{aligned}
\int_{D_{v}} a_{\mu}|\nabla v|^{p} d x & \leq \int_{D_{v}} a|\nabla v|^{p} d x+\int_{D_{v}}\left|a_{\mu}-a\right||\nabla v|^{p} d x \\
& \leq \int_{D_{u}} a|\nabla u|^{p} d x+\int_{B_{R}} a|\nabla \hat{v}|^{p} d x-\int_{B_{R}} a|\nabla u|^{p} d x+c(\mu-1) .
\end{aligned}
$$

By Proposition 1 and the definition of $s(M)$ we conclude that

$$
\int_{D_{v}} a_{\mu}|\nabla v|^{p} d x \leq s(M)+\int_{B_{R}} a\left(|\nabla \hat{v}|^{p}-|\nabla u|^{p}\right) d x+c\left|N_{u} \cap B_{R}\right| .
$$


for $R$ small enough. Thus (5.9) yields

$$
\begin{aligned}
s(M) \mu^{N-p-N p}\left(1+O\left(\left|N_{u} \cap B_{R}\right|\right)\right)^{p} & \leq s(M)+\int_{B_{R}} a\left(|\nabla \hat{v}|^{p}-|\nabla u|^{p}\right) d x \\
& +O\left(\left|N_{u} \cap B_{R}\right|\right),
\end{aligned}
$$

and rearranging terms we find for the expression

$$
I:=\int_{B_{R}} a\left(|\nabla u|^{p}-|\nabla \hat{v}|^{p}\right) d x
$$

the estimate

$$
I \leq\left(1-\mu^{N-p-N p}\right) \int_{D_{u}} a|\nabla u|^{p} d x+O\left(\left|N_{u} \cap B_{R}\right|\right)=O\left(\left|N_{u} \cap B_{R}\right|\right) .
$$

Let $u_{t}(x):=t u(x)+(1-t) \hat{v}(x)$ for $0 \leq t \leq 1$. Then we have

$$
\begin{aligned}
I & =\int_{B_{R}} a(x) \int_{0}^{1} \frac{d}{d t}\left|\nabla u_{t}\right|^{p} d t d x \\
& =p \int_{B_{R}} a(x) \int_{0}^{1}\left|\nabla u_{t}\right|^{p-2} \nabla u_{t} \cdot \nabla(u-\hat{v}) d t d x .
\end{aligned}
$$

Since $\hat{v} \geq u$

$$
\int_{B_{R}} a(x)|\nabla \hat{v}|^{p-2} \nabla \hat{v} \cdot \nabla(u-\hat{v}) d x=\lambda \int_{B_{R}} b(x)(\hat{v}-u) d x \geq 0,
$$

and thus

$$
I \geq p \int_{B_{R}} a(x) \int_{0}^{1}\left(\left|\nabla u_{t}\right|^{p-2} \nabla u_{t}-|\nabla \hat{v}|^{p-2} \nabla \hat{v}\right) \cdot \nabla(u-\hat{v}) d t d x .
$$

Replacing $u-\hat{v}$ by $\frac{1}{t}\left(u_{t}-\hat{v}\right)$ we get

$$
I \geq p \int_{0}^{1} \frac{1}{t} \int_{B_{R}} a(x)\left(\left|\nabla u_{t}\right|^{p-2} \nabla u_{t}-|\nabla \hat{v}|^{p-2} \nabla \hat{v}\right) \cdot \nabla\left(u_{t}-\hat{v}\right) d x d t .
$$

Now we use the following inequalities, which can be found e.g. in [12], Lemma 5.7

$\left(|\xi|^{p-2} \xi-\left|\xi^{\prime}\right|^{p-2} \xi^{\prime}\right) \cdot\left(\xi-\xi^{\prime}\right) \geq c(N, p)\left(|\xi|+\left|\xi^{\prime}\right|\right)^{p-2}\left|\xi-\xi^{\prime}\right|^{2} \quad$ if $\quad 1<p \leq 2$,

and

$$
\left(|\xi|^{p-2} \xi-\left|\xi^{\prime}\right|^{p-2} \xi^{\prime}\right) \cdot\left(\xi-\xi^{\prime}\right) \geq c(N, p)\left|\xi-\xi^{\prime}\right|^{p} \quad \text { if } \quad p \geq 2
$$

for all $\xi, \xi^{\prime} \in \mathbb{R}^{N}$.

Inserting the second inequality into (5.15) we get for $p \geq 2$ 


$$
\begin{aligned}
I & \geq c(N, p) p \int_{0}^{1} \frac{1}{t} \int_{B_{R}\left(x_{0}\right)} a(x)\left|\nabla\left(u_{t}-\hat{v}\right)\right|^{p} d x d t \\
& =c(N, p) p \int_{0}^{1} t^{p-1} d t \int_{B_{R}\left(x_{0}\right)} a(x)|\nabla(u-\hat{v})|^{p} d x \\
& =c(N, p) \int_{B_{R}\left(x_{0}\right)} a(x)|\nabla(u-\hat{v})|^{p} d x .
\end{aligned}
$$

From inequality (5.14) we deduce that

$$
\int_{B_{R}\left(x_{0}\right)} a(x)|\nabla(u-\hat{v})|^{p} d x \leq O\left(\left|N_{u} \cap B_{R}\left(x_{0}\right)\right|\right) .
$$

This proves the second assertion (5.7) of the lemma.

For the case $1<p \leq 2$ we have

$$
\begin{aligned}
I & \left.\geq c(N, p) p \int_{0}^{1} \frac{1}{t} \int_{B_{R}} a(x)\left|\nabla\left(u_{t}-\hat{v}\right)\right|^{2}\left(\left|\nabla u_{t}\right|+|\nabla \hat{v}|\right) \mid\right)^{p-2} d x d t \\
& \left.\geq c(N, p) \frac{p}{2} \int_{0}^{1} t d t \int_{B_{R}} a(x)|\nabla(u-\hat{v})|^{2}(|\nabla u|+|\nabla \hat{v}|) \mid\right)^{p-2} d x \\
& \left.=\frac{1}{4} c(N, p) \int_{B_{R}} a(x)|\nabla(u-\hat{v})|^{2}(|\nabla u|+|\nabla \hat{v}|) \mid\right)^{p-2} d x .
\end{aligned}
$$

We use Hölder's inequality and get

$$
\begin{aligned}
& \int_{B_{R}} a|\nabla(u-\hat{v})|^{p} d x \\
& \left.\left.=\int_{B_{R}} a^{\frac{p}{2}}|\nabla(u-\hat{v})|^{p}(|\nabla u|+|\nabla \hat{v}|) \mid\right)^{\frac{(p-2) p}{2}} a^{1-\frac{p}{2}}(|\nabla u|+|\nabla \hat{v}|) \mid\right)^{\frac{(2-p) p}{2}} d x \\
& \left.\left.\leq\left(\int_{B_{R}} a|\nabla(u-\hat{v})|^{2}(|\nabla u|+|\nabla \hat{v}|) \mid\right)^{p-2} d x\right)^{\frac{p}{2}}\left(\int_{B_{R}} a(|\nabla u|+|\nabla \hat{v}|) \mid\right)^{p} d x\right)^{1-\frac{p}{2}} .
\end{aligned}
$$

Rearranging terms gives:

$$
\begin{aligned}
& \left.\int_{B_{R}} a(x)|\nabla(u-\hat{v})|^{2}(|\nabla u|+|\nabla \hat{v}|) \mid\right)^{p-2} d x \\
& \geq 2^{1-\frac{2}{p}}\left(\int_{B_{R}} a|\nabla(u-\hat{v})|^{p} d x\right)^{\frac{2}{p}}\left(\int_{B_{R}} a|\nabla u|^{p} d x\right)^{1-\frac{2}{p}}
\end{aligned}
$$

where we have also used (5.4) Thus

$$
I \geq c(N, p)\left(\int_{B_{R}} a(x)|\nabla(u-\hat{v})|^{p} d x\right)^{\frac{2}{p}}\left(\int_{B_{R}} a(x)|\nabla u|^{p} d x\right)^{1-\frac{2}{p}}
$$


For the case $1<p \leq 2$ inequality (5.14) then implies

$$
\int_{B_{R}} a(x)|\nabla(u-\hat{v})|^{p} d x \leq c\left(\int_{B_{R}} a(x)|\nabla u|^{p} d x\right)^{1-\frac{p}{2}}\left|N_{u} \cap B_{R}\right|^{\frac{p}{2}}
$$

The integral $\int_{B_{R}} a(x)|\nabla u|^{p} d x$ can be estimated by means of a Caccioppoli type inequality, based on inequality (4.1), as follows

$$
\int_{B_{R}} a(x)|\nabla u|^{p} d x \leq \frac{c}{R^{p}} \int_{B_{2 R}} u^{p} d x \leq c|u|_{\infty} R^{N-p}
$$

where $c$ depends only on $a_{\min }, a_{\max }, b_{\max }, s(M)$ and $p$. For a derivation of the first inequality with a discussion of the optimal constant $c$ we refer to [17]. The second inequality in (5.17) uses Theorem 4. Thus

$$
\begin{aligned}
& \int_{B_{R}} a(x)|\nabla(u-\hat{v})|^{p} d x \\
& \leq c R^{(N-p)\left(1-\frac{p}{2}\right)}\left|N_{u} \cap B_{R}\right|^{\frac{p}{2}}
\end{aligned}
$$

for $1<p \leq 2$. This completes the proof of the lemma.

After this preparation we are in position to proceed, as in [19], to the proof of the Theorem 5 .

Proof of Theorem 5 We use the setting as given by the previous lemmas. For $r<R$ and $1<p<\infty$ we estimate

$$
\int_{B_{r}\left(x_{0}\right)} a(x)|\nabla u|^{p} d x \leq \int_{B_{R}\left(x_{0}\right)} a(x)|\nabla(u-\hat{v})|^{p} d x+\int_{B_{r}\left(x_{0}\right)} a(x)|\nabla \hat{v}|^{p} d x .
$$

The first term on the right-hand side can be estimated by Lemma 8 . In order to estimate the last term we use the regularity result of [9] which states that $\hat{v} \in C^{1, \alpha}\left(\bar{B}_{\rho}\left(x_{0}\right)\right)$ for any fixed $\rho<R$. Hence

$$
\int_{B_{r}\left(x_{0}\right)} a(x)|\nabla \hat{v}|^{p} d x \leq c r^{N}
$$

where $c$ depends only on $\rho$ and not on $r \leq \rho$. Next we consider the auxiliary function $h$, defined as the unique solution of

$$
\begin{aligned}
& \operatorname{div}\left(a(x)|\nabla h|^{p-2} \nabla h\right)=0 \quad \text { in } \quad B_{R}\left(x_{0}\right) \\
& h=u \quad \text { in } \quad \partial B_{R}\left(x_{0}\right)
\end{aligned}
$$

Thus we have by the minimality of $h$

$$
\int_{B_{R}\left(x_{0}\right)} a(x)|\nabla u|^{p} d x \geq \int_{B_{R}\left(x_{0}\right)} a(x)|\nabla h|^{p} d x=: c_{1} .
$$


Thus we obtain

$$
\begin{array}{r}
\int_{B_{r}\left(x_{0}\right)} a(x)|\nabla \hat{v}|^{p} d x \leq \frac{c r^{N}}{c_{1}} \int_{B_{R}} a(x)|\nabla h|^{p} d x \\
\leq c\left(N, p, a_{\min }, a_{\max }\right)\left(\frac{r}{R}\right)^{N} \int_{B_{R}\left(x_{0}\right)} a(x)|\nabla u|^{p} d x .
\end{array}
$$

Taking into account (5.6) and (5.7) we arrive at

$$
\int_{B_{r}\left(x_{0}\right)}|\nabla u|^{p} d x \leq c\left(N, p, a_{\min }, a_{\max }\right)\left(\frac{r}{R}\right)^{N} \int_{B_{R}\left(x_{0}\right)}|\nabla u|^{p} d x+c R^{N-p+\frac{p}{2} p} .
$$

for $1<p<2$ and

$$
\int_{B_{r}\left(x_{0}\right)}|\nabla u|^{p} d x \leq c\left(N, p, a_{\min }, a_{\max }\right)\left(\frac{r}{R}\right)^{N} \int_{B_{R}\left(x_{0}\right)}|\nabla u|^{p} d x+c R^{N},
$$

for $p \geq 2$.

Now we apply Lemma 7 and we obtain in the first case $1<p<2$

$$
\int_{B_{r}\left(x_{0}\right)}|\nabla u|^{p} d x \leq C\left(\frac{r}{R}\right)^{\tilde{\beta}}\left[\int_{B_{R}\left(x_{0}\right)}|\nabla u|^{p} d x+\text { const. } R^{\tilde{\beta}}\right]
$$

with $\tilde{\beta}=N-p+\frac{p}{2} p$ and we set $\beta=\frac{p}{2}$. We apply Lemma 6 and get $u \in C_{l o c}^{0, \beta}$ for all $0 \leq \beta \leq \frac{p}{2}$. We easily extend this result to all $0<\beta<1$. Indeed if we set $\beta_{1}=\frac{p}{2}$ we can use the fact that $u \in C_{l o c}^{0, \beta_{1}}$ in inequality (5.17) and obtain a bound with a higher power in $R$. Following the same procedure then gives $u \in C_{l o c}^{0, \beta_{2}}$ with $\beta_{2}>\beta_{1}$. After finitely many repetitions we obtain $u \in C_{l o c}^{0, \beta}$ for any $0<\beta<1$.

For $p \geq 2$ we use (5.6) and derive (5.20) for any $0 \leq \tilde{\beta}<N$. We write $\tilde{\beta}$ as $\tilde{\beta}=N-p+\beta p$ for any $0<\beta<1$. The assertion now follows from Morrey's Dirichlet growth theorem.

Based on this we now prove the Lipschitz continuity of any minimizer.

Theorem 6 Assume (A1)-(A4) and $p \geq 2$. Let $u \in \mathcal{K}$ be a minimizer of $s(M)$. Then $u \in C_{l o c}^{0,1}(B)$.

Proof The proof follows closely the proof of theorem 2.3 in [2]. Set $d(x):=$ $\operatorname{dist}\left(x, N_{u}\right)$. Since $u$ is continuous the set $D_{u}$ is open. We will use (5.7):

$$
\int_{B_{R}\left(x_{0}\right)}|\nabla(u-\hat{v})|^{p} d x \leq c\left|N_{u} \cap B_{R}\left(x_{0}\right)\right| .
$$

Let $x_{0}$ be any point in $B$ be such that $d\left(x_{0}\right)<\frac{1}{2} \operatorname{dist}\left(x_{0}, \partial B\right)$. 


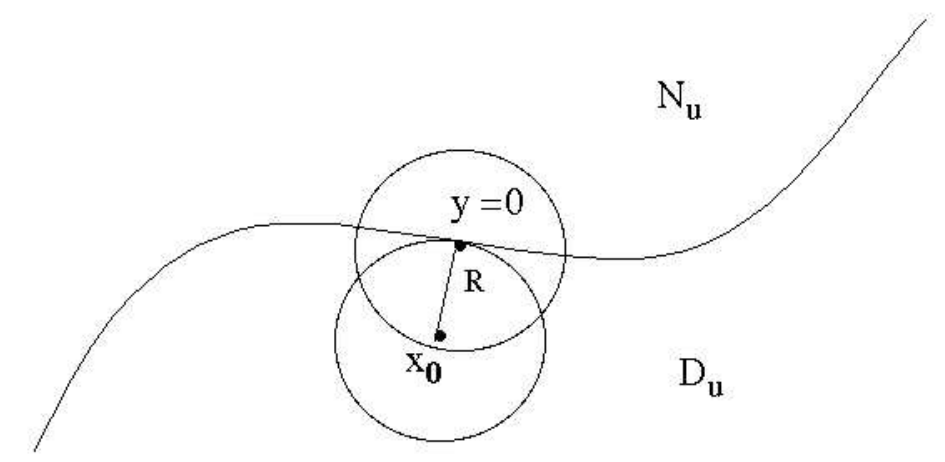

Figure 1

We prove, that the estimate $u\left(x_{0}\right) \leq c d\left(x_{0}\right)$ must hold for some positive constant $c$ which does not depend on $x_{0}$. The idea of the proof is to assume that there exists a positive number $M$ independent of $x_{0}$ such that

$$
u\left(x_{0}\right)>M d\left(x_{0}\right)
$$

and then to derive an upper bound for $M$. This implies that there is a constant $M^{\prime}$ such that $u\left(x_{0}\right) \leq M^{\prime} d\left(x_{0}\right)$. Let $R=d\left(x_{0}\right)$ and consider the ball $B_{R}\left(x_{0}\right)$. It is contained in $D_{u}$. Since

$$
\operatorname{div}\left(a(x)|\nabla u(x)|^{p-2} \nabla u(x)\right)+\lambda b(x)=0 \quad \text { in } B_{R}\left(x_{0}\right) \subset \bar{D}_{u},
$$

we can apply Harnack's inequality cf. e.g. [10] and we have

$$
\inf _{B_{\frac{3}{4} R}\left(x_{0}\right)} u \geq c u\left(x_{0}\right)>c M R .
$$

by (5.21). Since $R=d\left(x_{0}\right)$ the boundary $\partial B_{R}\left(x_{0}\right)$ touches $N_{u}$ in at least one point. Let $y \in \partial B_{R}\left(x_{0}\right) \cap N_{u}$. After translation we may assume that $y=0$. Next we consider the ball $B_{R}(0)$. Let $\hat{v}$ the solution to

$$
\begin{array}{rll}
\operatorname{div}\left(a(x)|\nabla \hat{v}|^{p-2} \nabla \hat{v}\right)+\lambda b(x)=0 & \text { in } & B_{R}(0) \\
\hat{v}=u & \text { in } & \partial B_{R}(0)
\end{array}
$$

This is the same function as in (5.3). Thus $\hat{v} \geq u$ in $B_{R}(0)$ and (5.7) holds. From (5.23) we deduce

$$
\hat{v}(x) \geq c M R \quad \text { in } B_{\frac{3}{4} R}\left(x_{0}\right) \cap B_{R}(0) .
$$


We apply Harnack's inequality once more and get

$$
\hat{v}(x) \geq C^{*} \quad \text { in } B_{\frac{1}{2} R}(0)
$$

with $C^{*}=c M R$. We introduce the function

$$
w(x):=C^{*}\left(e^{-\mu|x|^{2}}-e^{-\mu R^{2}}\right)
$$

Direct computation gives

$$
\operatorname{div}\left(a(x)|\nabla w(x)|^{p-2} \nabla w(x)\right)+b(x)>0 \quad \text { in } \quad B_{R} \backslash B_{\frac{1}{2} R}(0)
$$

if $\mu$ is sufficiently large. (This is only true for $p \geq 2$.) Since $w=0$ in $\partial B_{R}$ we get

$$
w \leq C^{*} \leq \hat{v} \quad \text { in } \quad \partial B_{\frac{1}{2} R}(0)
$$

The maximum principle then implies

$$
\hat{v}(x) \geq w(x) \geq c C^{*}(R-|x|) \quad \text { in } \quad B_{R} \backslash B_{\frac{1}{2} R}(0) .
$$

(5.25) then implies

$$
\hat{v}(x) \geq c M(R-|x|) \quad \text { in } B_{R}(0)
$$

With exactly the same arguments as in [1] Lemma 3.2 we now derive from (5.27) the inequality

$$
c M|S| \leq \int_{S}|\nabla(u-\hat{v})| d x
$$

for some set $S$ in $B_{R}(0)$, which contains $N_{u} \cap B_{R}(0)$. This implies

$$
c M|S| \leq \int_{S}|\nabla(u-\hat{v})| d x \leq|S|^{1-\frac{1}{p}}\left(\int_{S}|\nabla(u-\hat{v})|^{p} d x\right)^{\frac{1}{p}} .
$$

Hence

$$
M^{p}|S| \leq \int_{B_{R}(0)}|\nabla(u-\hat{v})|^{p} d x \leq c\left|N_{u} \cap B_{R}(0)\right|
$$

and this gives an upper bound for $M$. Hence we proved

$$
u(x) \leq c d(x)
$$

for some positive constant $c$. Together with interior regularity estimates in $D_{u}$ we obtain the result.

Acknowledgement The paper was completed while the first author (C.B) was visiting the Department of Mathematics of the RWTH. She would like to express her gratitude for the hospitality and the stimulating atmosphere. 


\section{References}

[1] H. W. Alt and L. A. Caffarelli, Existence and regularity for a minimum problem with free boundary, J. reine angew. Math. 325 (1981), 105-144.

[2] H. W. Alt, L. A. Caffarelli and A. Friedman, Variational problems with two phases and their free boundaries, Trans. AMS 2 (1984), 431-461.

[3] C. Bandle, Isoperimetric inequalities and applications, Pitman (1980).

[4] C. Bandle, Sobolev inequalities and quasilinear boundary value problems, Basel Preprint 2001-02, Nonlinear Analysis and Applications: To V. Lakshmikantham on his 80th Birthday, R.P. Agarwal, D O'Regan eds., Vol. 1, Kluwer (2003), 227-240.

[5] C. Bandle and A. Wagner, A Sobolev constant in multiply connected domains, to appear.

[6] T. Briançon, M. Hayouni and M. Pierre, Lipschitz continuity of state functions in some optimal shaping, Calc. Var. Partial Differential Equations 23 (2005), no. 1, 13-32.

[7] F. Catrina and Z. Q. Wang, Positive bound states having prescribed symmetry for a class of nonlinear elliptic equations in $\mathbb{R}^{N}$, Ann. Inst. $\mathrm{H}$. Poincaré 18 (2001), 157-178.

[8] J. I. Diaz, Nonlinear partial differential equations and free boundaries, Pitman Research Notes in Mathematics, 106 (1985).

[9] E. DiBenedetto, $C^{1+\alpha}$ local regularity of weak solutions of degenerate elliptic equations, Nonlinear Analysis, 7 (1983), 827-850.

[10] P. Drábek, A. Kufner and F. Nicolosi, Quasilinear elliptic equations with degenerations and singularities, De Gruyter, Series in Nonlinear Analysis and Applications, 5 (1991).

[11] M. Giaquinta, Multiple integrals in the calculus of variations and nonlinear elliptic systems, Ann. Math. Studies 105, Princeton University Press (1983).

[12] J.Maly, W.P. Ziemer, Fine Regularity of Solutions of Elliptic Partial Differential Equations, Mathematical Surveys and Monographs, Volume 51, AMS (1997),

[13] V.G. Maz'ja, Sobolev spaces, Springer (1985).

[14] C. B. Morrey, Multiple integrals in the calculus of variations, Springer, Heidelberg, New York (1966).

[15] G. Pólya, G. Szegö, Isoperimetric Inequalities in Mathematical Physics, Princeton University Press (1951).

[16] B. Opic and A. Kufner, Hardy-type inequalities, Longman, Research Notes 219 (1990).

[17] I. Peric and D. Zubrinic, Caccioppoli's inequality for quasilinear elliptic operators, Math. Inequal. Appl. 2, no. 2, 251-261, (1999). 
[18] J-L. Vazquez, A strong maximum principle for some quasilinear elliptic equations, Appl. Math. and Optimization 12 (1984), 191-202.

[19] A. Wagner, Optimal shape problems for eigenvalues, Comm. PDE. Volume 30 (7-9), (2005). 